\title{
Analysis of Heat and Mass Transfer in a Porous Cavity Containing Water near Its Density Maximum
}

\author{
Murugan Muthtamilselvan \\ Department of Applied Mathematics, Bharathiar University, Coimbatore, India \\ E-mail:muthtamil1@yahoo.co.in \\ Received September 23, 2010; revised May 31, 2011; accepted June 7, 2011
}

\begin{abstract}
A numerical study is performed on steady natural convection inside a porous cavity with cooling from the side walls. The governing equations are solved by finite volume method. Representative results illustrating the effects of the thermal Rayleigh number, density inversion parameter, buoyancy ratio and Schmidt number on the contour maps of the fluid flow, temperature and concentration are reported. It is found that the number of cells which form in the cavity varies primarily with the density inversion parameter and is always even due to the symmetry imposed by the cold sidewalls. In addition that the flow becomes weaker as the Darcy number decreases from the pure fluid limit towards the Darcy-flow limit.
\end{abstract}

Keywords: Convection, Double Diffusive, Porous Medium, Finite Volume Method

\section{Introduction}

Natural convection in enclosures containing a fluidsaturated porous medium has received much attention recently because the data concerning buoyant enclosure flow are in great demand for many traditional and contemporary applications such as insulating systems for buildings and heat exchanger devices, energy storage systems, material processing and geothermal systems $[1,2]$. Another reason leading to study of the convection in porous medium enclosures is the interest in fundamental phenomena of double diffusive and density of the convective flow. A comprehensive review of the literature on double diffusion, natural convection in saturated porous media may be found in [3-6].

Convection in water behaves differently around the temperature region of $4^{\circ} \mathrm{C}$ due to the anomalous behavior of density around this temperature and the density of water at both side walls of the cavity various linearly with the temperature. A number of studies have investigated the effect of the density extremum for water in detail. Nansteel et al. [7] studied the natural convection of cold water in the vicinity of its density maximum in a rectangular enclosure in the limit of small Rayleigh number. They observed that the strength of the counter rotating flow decreases with decreasing aspect ratio. Mahidjiba et al. [8] investigated onset of convection in a horizontal anisotropic porous layer saturated with water near $4^{\circ} \mathrm{C}$. It is found that the onset of motion dependent permeability ratio and inversion parameter. The Brinkman's extension of Darcy's law has been used in a study by Bannacer et al. [9] to investigate double diffusive convection in anisotropic porous media with high porosity. It is demonstrated that the anisotropic properties of the porous medium considerably modify the heat and mass transfer rates from that expected under isotropic conditions.

Very recently, Muthtamilselvan et al. [10] studied numerically convection in a two-sided lid-driven heat generating porous cavity with alternative thermal boundary conditions. They found that the variation of the average Nusselt number is non-linear for increasing values of the Darcy number with uniform and non-uniform heating condition. The aim of the present study a double diffusive natural convection flow in a square cavity filled with a fluid saturated porous medium when the bottom wall is heated.

\section{Mathematical Analysis}

Consider a steady-state two-dimensional square cavity filled with a porous medium of length $\mathrm{L}$ as shown in Figure 1. Different temperature and concentrations are imposed between the bottom $\left(\theta_{h}, c_{h}\right)$ and vertical side walls $\left(\theta_{c}, c_{c}\right)$, where the $\theta_{h}>\theta_{c}$ and $c_{h}>c_{c}$. The top wall is considered to be adiabatic. The physical proper- 


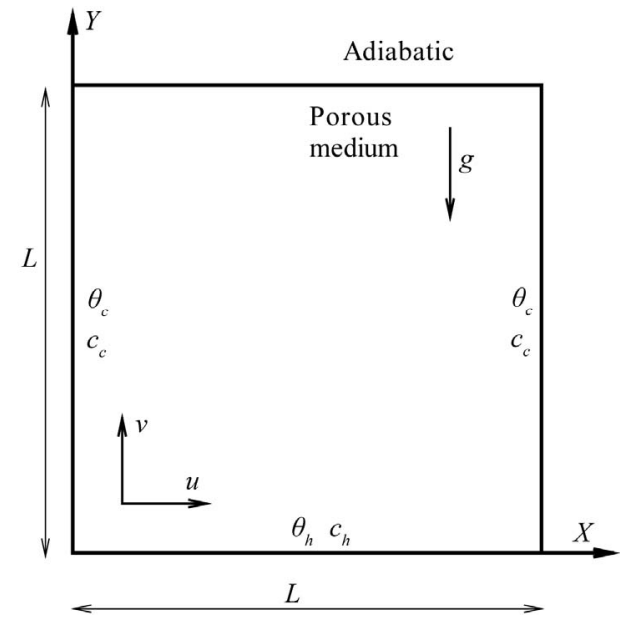

Figure 1. Flow configuration and coordinate system.

ties are considered to be constant except the density variation in the body force term of the momentum equation which is satisfied by the Boussinesq's approximation. The density of the cold water is assumed to vary with temperature according to the following equation

$$
\begin{gathered}
\rho=\rho_{0}\left[1-\beta_{T}\left(\theta-\theta_{1}\right)^{2}+\beta_{c}\left(c-c_{0}\right)\right] \\
\left(\beta_{T}=8.0 \times 10^{-6}\left({ }^{\circ} \mathrm{C}\right) K^{-2}, \beta_{c}=3.0 \times 10^{-3} \mathrm{~m}^{3} \mathrm{~kg}^{-1}\right)
\end{gathered}
$$

where $\beta_{T}$ and $\beta_{c}$ are the coefficients for thermal and concentration expansions. In the present investigation the porous medium is assumed to be hydrodynamically and thermally isotropic and saturated with a fluid that is in local thermal equilibrium (LTE) with the solid matrix. A general Brinkman-Forchheimer extended Darcy model is used to account for the flow in the porous medium. Using the above assumptions, the governing equations for mass, momentum and energy can be written in the dimensional form as

$$
\begin{gathered}
\frac{\partial u}{\partial x}+\frac{\partial v}{\partial y}=0 \\
\frac{1}{\varepsilon^{2}}\left(u \frac{\partial u}{\partial x}+v \frac{\partial u}{\partial y}\right) \\
=-\frac{1}{\rho_{0}} \frac{\partial p}{\partial x}+\frac{v}{\varepsilon} \nabla^{2} u-\frac{v}{K} u-\frac{1.75}{\sqrt{150} \varepsilon^{3 / 2}} \frac{u\left(u^{2}+v^{2}\right)^{1 / 2}}{\sqrt{K}} \\
\frac{1}{\varepsilon^{2}}\left(u \frac{\partial v}{\partial x}+v \frac{\partial v}{\partial y}\right) \\
=-\frac{1}{\rho_{0}} \frac{\partial p}{\partial y}+\frac{v}{\varepsilon} \nabla^{2} v-\frac{v}{K} v-\frac{1.75}{\sqrt{150} \varepsilon^{3 / 2}} \frac{v\left(u^{2}+v^{2}\right)^{1 / 2}}{\sqrt{K}}-\frac{\rho}{\rho_{0}} g
\end{gathered}
$$

$$
\begin{aligned}
& u \frac{\partial \theta}{\partial x}+v \frac{\partial \theta}{\partial y}=\alpha \nabla^{2} \theta \\
& u \frac{\partial c}{\partial x}+v \frac{\partial c}{\partial y}=D \nabla^{2} c
\end{aligned}
$$

The appropriate initial and boundary conditions are:

$$
\begin{aligned}
& t=0: u=v=0, \theta=\theta_{c}, c=c_{c}, 0 \leq x \leq 1,0 \leq y \leq 1 \\
& t>0: u=v=0, \frac{\partial \theta}{\partial y}=\frac{\partial c}{\partial y}=0,0 \leq x \leq 1, y=1 \\
& u=v=0, \theta=\theta_{h}, c=c_{h}, y=0, \quad 0 \leq x \leq 1 \\
& u=v=0, \theta=\theta_{c}, c=c_{c}, x=0 \& 1, \quad 0 \leq y \leq 1
\end{aligned}
$$

Dimensionless variables are defined as follows:

$$
\begin{aligned}
& (X, Y)=\frac{(x, y)}{L},(U, V)=\frac{(u, v)}{v / L}, T=\frac{\theta-\theta_{c}}{\theta_{h}-\theta_{c}}, \\
& C=\frac{c-c_{c}}{c_{h}-c_{c}}, \text { with } \theta_{h}>\theta_{c}, c_{h}>c_{c} \text { and } P=\frac{p L^{2}}{\rho_{0} v^{2}}
\end{aligned}
$$

The non-dimensional form of the Equations (1)-(5) is obtained as:

$$
\frac{\partial U}{\partial X}+\frac{\partial V}{\partial Y}=0
$$

$$
\begin{aligned}
& \frac{1}{\varepsilon^{2}}\left(U \frac{\partial U}{\partial X}+V \frac{\partial U}{\partial Y}\right) \\
& =-\frac{\partial P}{\partial X}+\frac{\operatorname{Pr}}{\varepsilon} \nabla^{2} U-\frac{\operatorname{Pr}}{D a} U-\frac{1.75}{\sqrt{150} \sqrt{D a}} \frac{U\left(U^{2}+V^{2}\right)^{1 / 2}}{\varepsilon^{3 / 2}}
\end{aligned}
$$

$$
\begin{array}{r}
\frac{1}{\varepsilon^{2}}\left(U \frac{\partial V}{\partial X}+V \frac{\partial V}{\partial Y}\right) \\
=-\frac{\partial P}{\partial Y}+\frac{\operatorname{Pr}}{\varepsilon} \nabla^{2} V-\frac{\operatorname{Pr}}{D a} V-\frac{1.75}{\sqrt{150} \sqrt{D a}} \frac{V}{+\frac{R a_{T}}{\operatorname{Pr}}\left[(T-R)^{2}-N C\right]} \\
U \frac{\partial T}{\partial X}+V \frac{\partial T}{\partial Y}=\frac{1}{\operatorname{Pr}} \nabla^{2} T \\
U \frac{\partial C}{\partial X}+V \frac{\partial C}{\partial Y}=\frac{1}{S c} \nabla^{2} C
\end{array}
$$$$
=-\frac{\partial P}{\partial Y}+\frac{\operatorname{Pr}}{\varepsilon} \nabla^{2} V-\frac{\operatorname{Pr}}{D a} V-\frac{1.75}{\sqrt{150} \sqrt{D a}} \frac{V\left(U^{2}+V^{2}\right)^{1 / 2}}{\varepsilon^{3 / 2}}
$$

The initial and boundary conditions in dimensionaless form are:

$$
\tau=0: U=V=0, C=T=0,0 \leq X \leq 1,0 \leq Y \leq 1
$$




$$
\begin{aligned}
& \tau>0: U=V=0, \frac{\partial T}{\partial Y}=\frac{\partial C}{\partial X}=0,0 \leq X \leq 1, Y=1 \\
& U=V=0, C=1, T=1, Y=0,0 \leq X \leq 1 \\
& U=V=0, C=0, T=0, X=0 \& 1,0 \leq Y \leq 1
\end{aligned}
$$

The dimensionless variables are defined as: $D a=\frac{K}{H^{2}}$, Darcy number, $\operatorname{Pr}=\frac{v}{\alpha}, \operatorname{Pr}$ andtl number, $S c=\frac{v}{D}$, Schmidt number, $R a_{T}=\frac{g \beta T\left(\theta_{h}-\theta_{c}\right)^{2} L^{3}}{v^{2}}$, Solutal Rayleigh number, $R=\frac{\theta_{1}-\theta_{c}}{\theta_{h}-\theta_{c}}$, density inversion parameter, $N=\frac{R a_{c}}{R a_{T}}$, Buoyancy ratio number.

The average Nusselt number and Sherwood number is

$$
N u_{\text {avg }}=\left.\int_{0}^{1} \frac{\partial T}{\partial Y}\right|_{Y=0} \mathrm{~d} X, S c_{\text {avg }}=\left.\int_{0}^{1} \frac{\partial C}{\partial Y}\right|_{Y=0} \mathrm{~d} X
$$

\section{Method of Solution}

The governing equations along with the boundary conditions are solved numerically employing finite volume method using staggered grid arrangement. The semiimplicit method for pressure linked equation (SIMPLE) is used to couple momentum and continuity equations as given by Patankar [11]. The third order accurate deferred QUICK scheme of Hayase et al. [12] is employed to minimize the numerical diffusion for the convective terms for both the momentum equations and energy equation. The solution of the discretized momentum and pressure correction equations is obtained by TDMA line-by-line method. For complete details and code validation, the author is referred to the prior publication [10, 13]. The grid independence test is performed using successively sized uniform grids, $21 \times 21$ to $91 \times 91$. After grid independence check considering the accuracy and the computational time, all the computations are performed with a $41 \times 41$ grid.

\section{Results and Discussion}

Computations have been carried out for various values of the Darcy number $\left(\mathrm{Da}=10^{-4}-10^{-1}\right)$, the thermal Rayleigh number $\left(R a_{T}=10^{2}-10^{5}\right)$, the buoyancy ratio number $(N=0.2-0.8)$, the density inversion parameter $(R=0.1-0.3)$, the Schmidt number $(S c=5-50)$ and fixed value of $\operatorname{Pr}=11.573$ and porosity $(\varepsilon=0.4)$. The results are presented as streamlines, isotherms and isoconcentrations. The rate of heat and mass transfer in the enclosure is measured in terms of the average Nusselt and Sherwood numbers.

Figure 2 illustrates the streamlines, isotherms and isoconcentration of the numerical results for various thermal Rayleigh number. When $R a_{T}=10^{2}$ the flow is seen to be very weak as observed from streamlines. Therefore, the temperature and isoconcentration distributions are similar to that with stationary fluid and the heat transfer is due to purely conduction. When $R a_{T}=10^{3}$, streamline show the major cells occupied entire cavity and the minor cells are visible near bottom corners of the cavity. During conduction dominant mass transfer, the isoconcentration contours with $c=0.06$ occur symmetrically near the side walls of the cavity. The other isoconcentration contours with $c \geq 0.13$ are smooth curves which span the entire cavity and they are generally symmetric with respect to the vertical symmetric line. As thermal Rayleigh number increases from $10^{4}$ to $10^{5}$ streamline shows that the bottom corner minor cells are reduced its strength and size. The corresponding temperature contour illustrates the smooth cure bended towards bottom of the cavity. It should be noted in Figure 2, with increasing $R a_{T}$ from $10^{2}$ to $10^{4}$ the minor cells grow its size and reduced when $R a_{T}=10^{5}$. It is observed that the conduction heat transfer switches to convection heat transfer with increasing $R a_{T}$ from $10^{2}$ to $10^{4}$. Again the conduction heat transfer dominates when $R a_{T}=10^{5}$.

Streamline, isotherm and isoconcentration contours are displayed in Figure 3, for different values of Schmidt number. The streamline shows the minor cells are occupied top corners of the cavity and it increases its size and strength when Sc increases. The circulations are greater near the center and least at the wall due to no slip boundary conditions. The greater circulation in each half of the box follows a progressive wrapping around the centers of rotation and a more pronounced compression of the isotherms toward the boundary surfaces of the cavity occurs. Due to greater circulations near the central core at the top half of the cavity, there are small gradients in temperature whereas a large stratification zone of temperature is observed at the vertical symmetry line due to stagnation of flow.

Figure 4 shows the average Nusselt and Sherwood numbers for different density inversion parameter with different Darcy number. Decreasing of Darcy number decreased the heat and mass transfer rate. In such situation the heat transfer is dominated by conduction. Figure 5 illustrate that the average Nusselt and Sherwood number for thermal Rayleigh number Vs density inversion parameter. Decreasing of thermal Rayleigh number decreased the heat and mass transfer rate. Figure 6 shows the behavior of the average Nusselt and Sherwood number for different $\mathrm{N}$ Vs Sc. It is found that the average Nusselt and Sherwood number gets minimum in the den- 

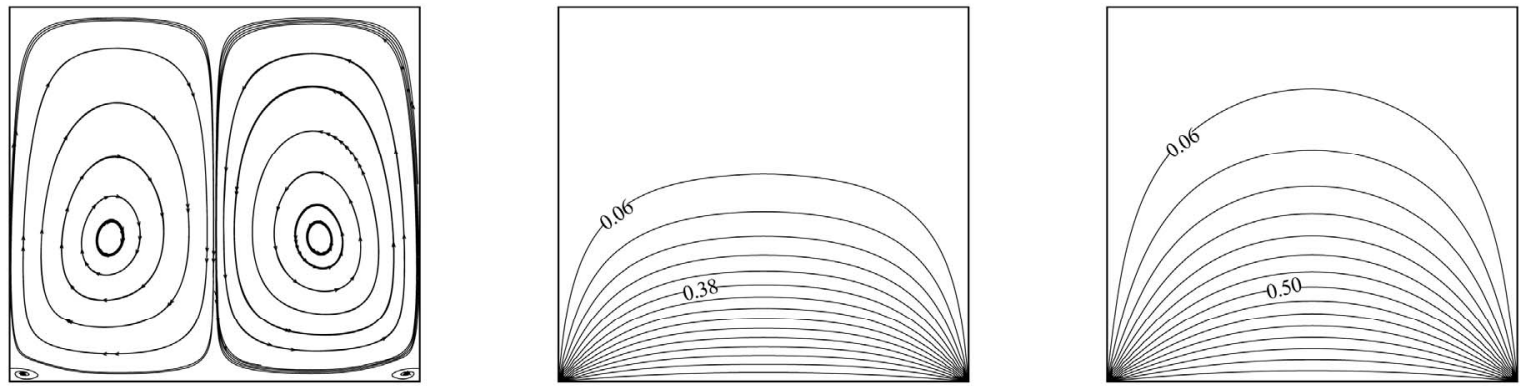

(a) $R a_{T}=10^{2}$
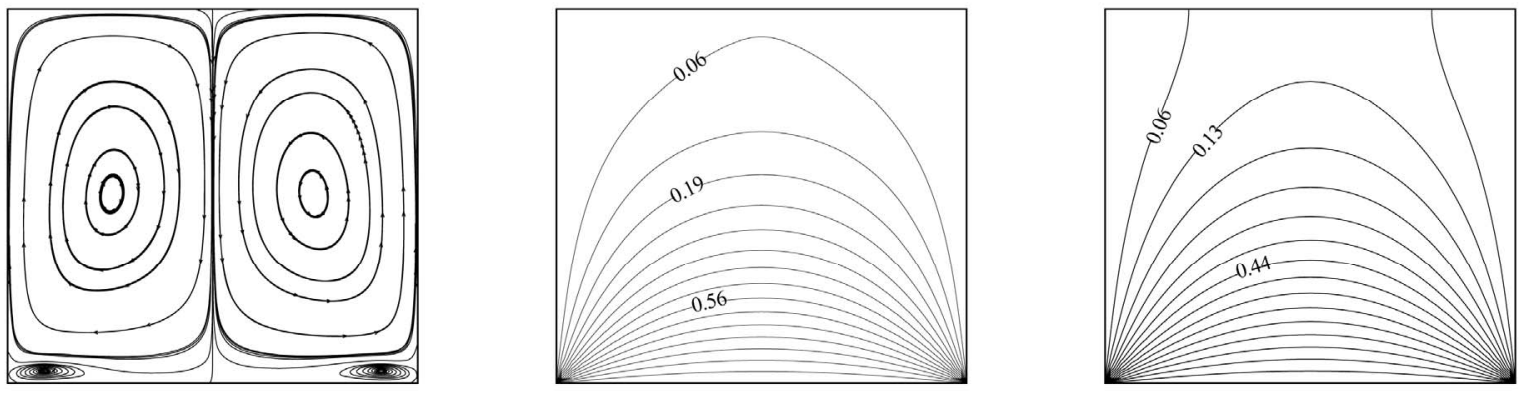

(b) $R a_{T}=10^{3}$
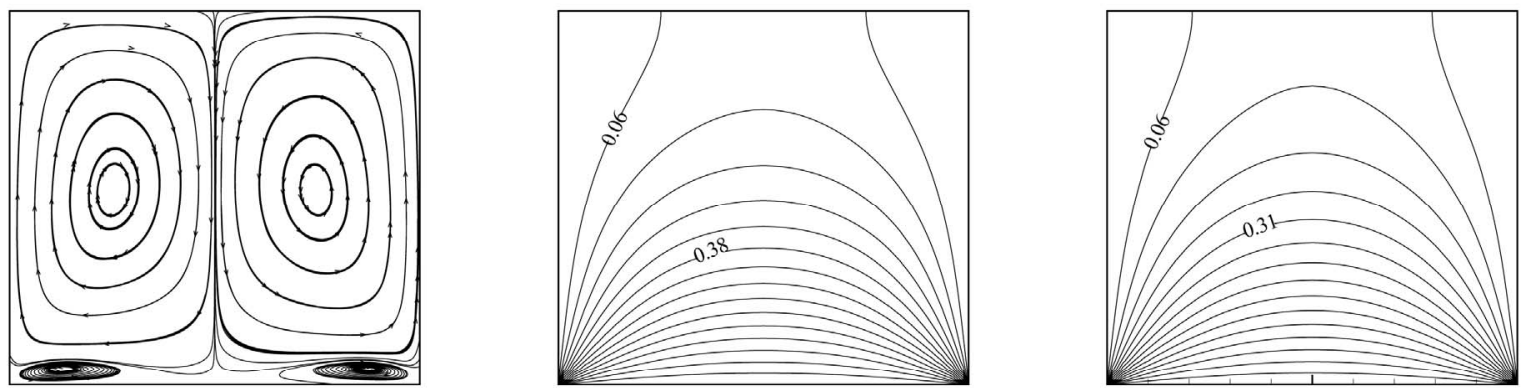

(c) $R a_{T}=10^{4}$
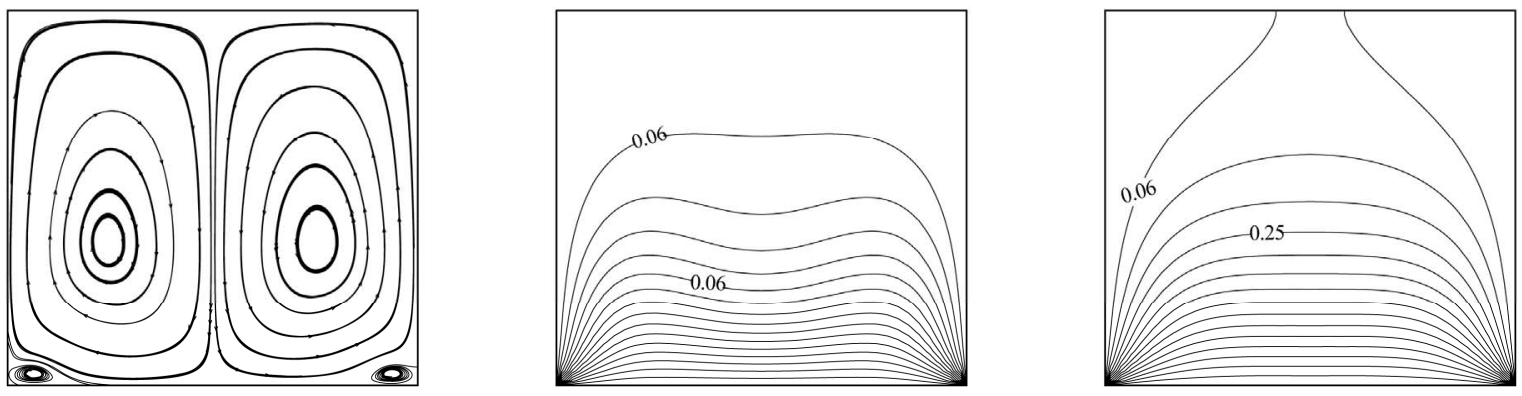

(d) $R a_{T}=10^{5}$

Figure 2. Streamlines, isotherms, iso-concentration for different $\operatorname{Ra}_{T}, \mathrm{Da}=10^{-2}, N=0.5, S c=5, R=0.2$. 

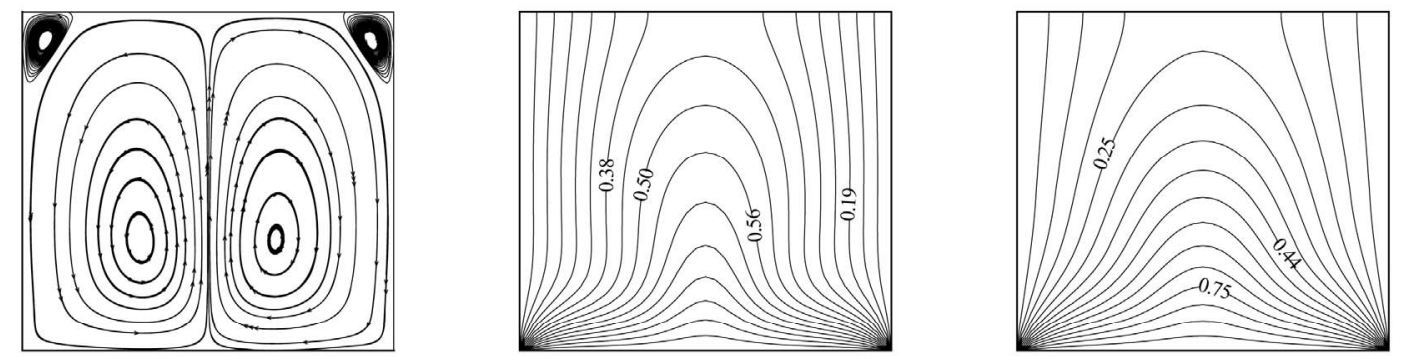

(a) $S_{c}=5$
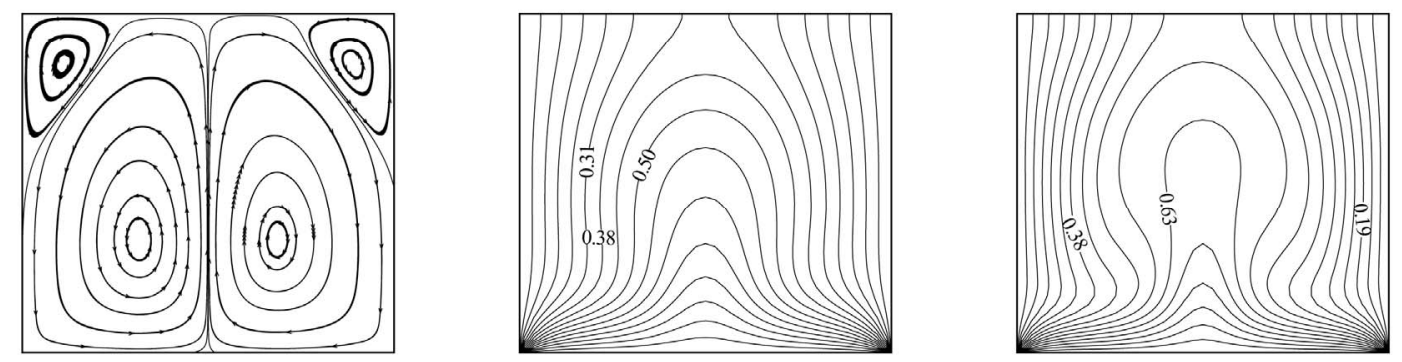

(b) $S_{c}=25$
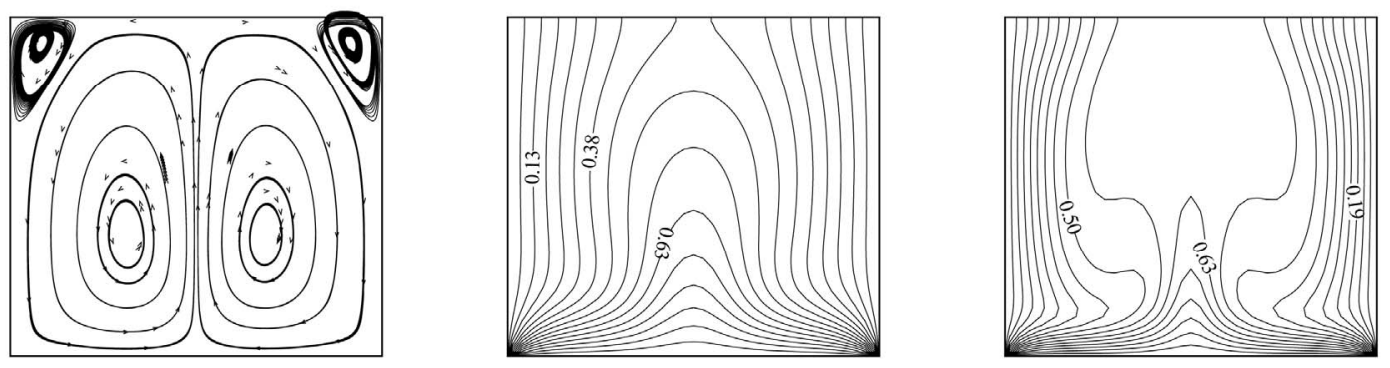

(c) $S_{c}=50$

Figure 3. Streamlines, isotherms, iso-concentration for different $S c, D a=10^{-2}, N=0.2, R_{T}=10^{5}$.
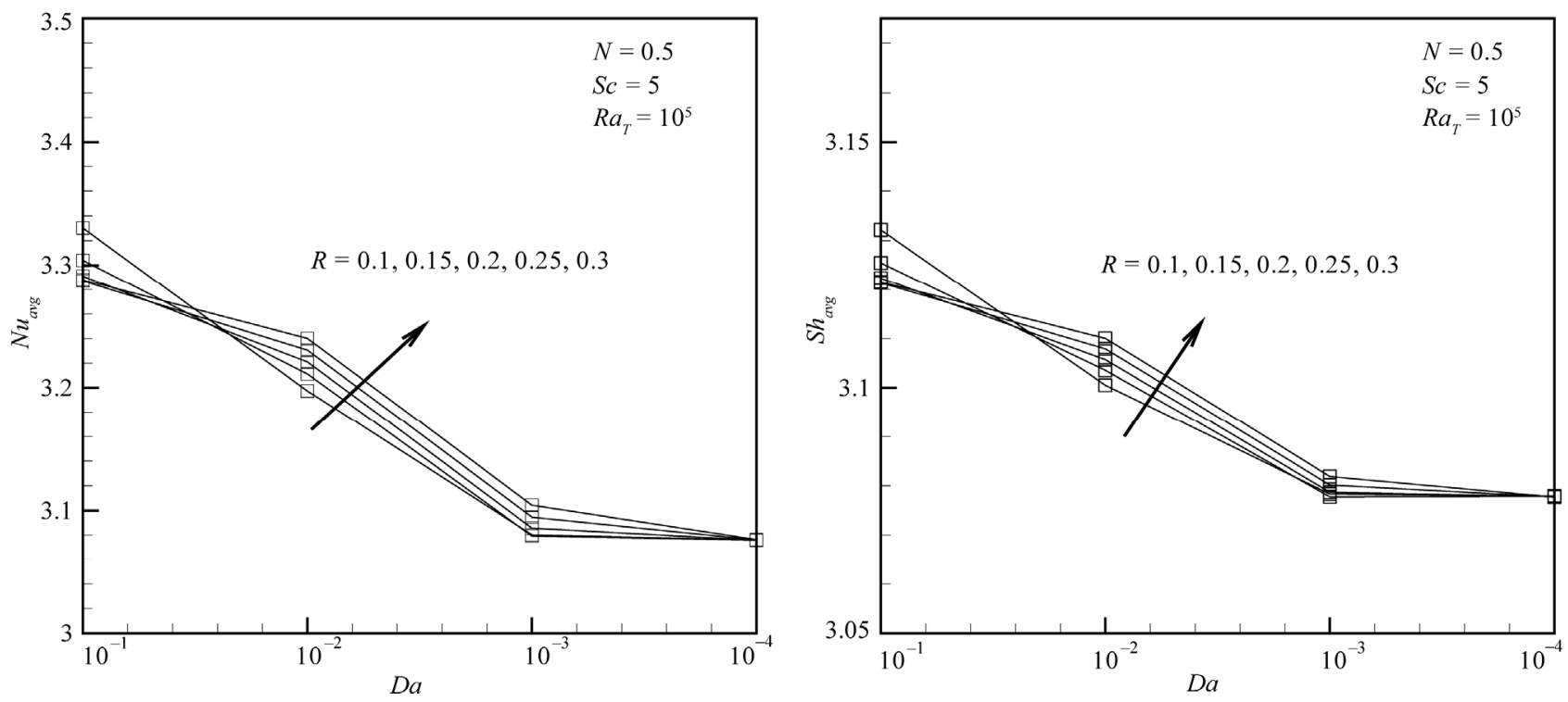

Figure 4. Average Nusselt number and Sherwood number for $\mathrm{Da}$ Vs $R$. 

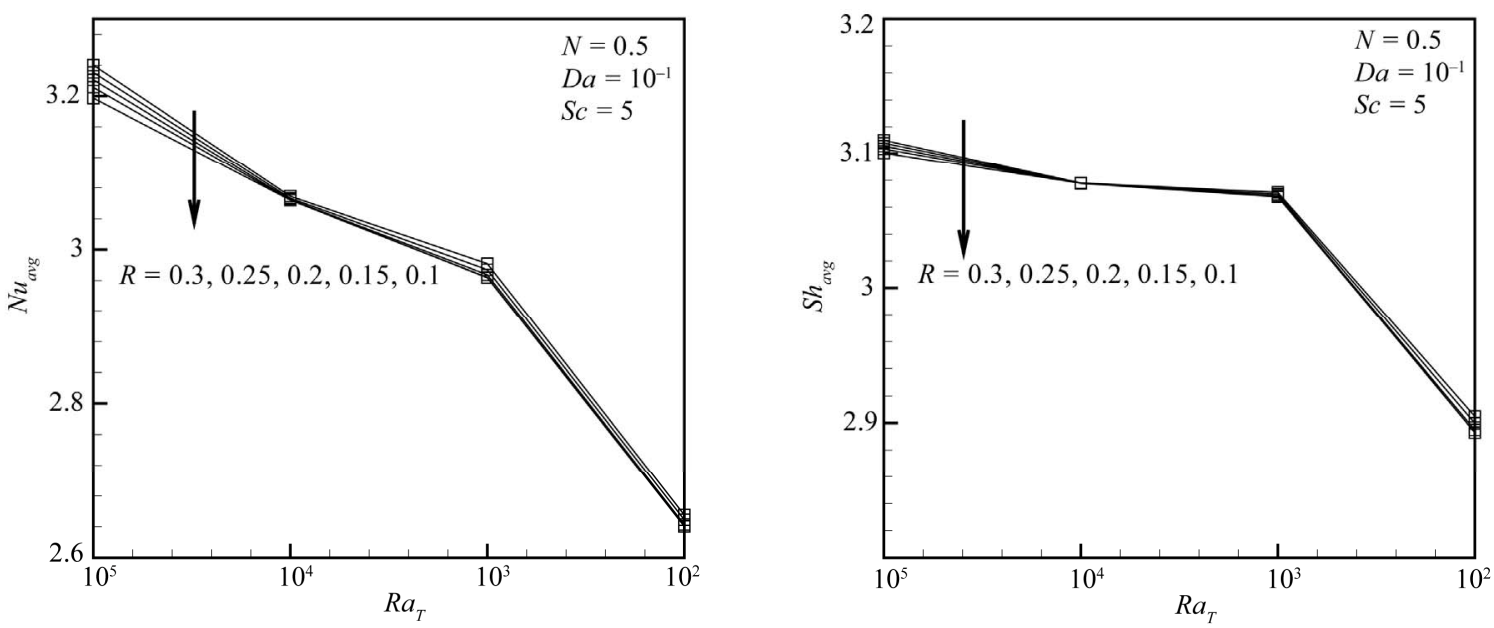

Figure 5. Average Nusselt number and Sherwood number for $R a_{T} \operatorname{Vs} R$.
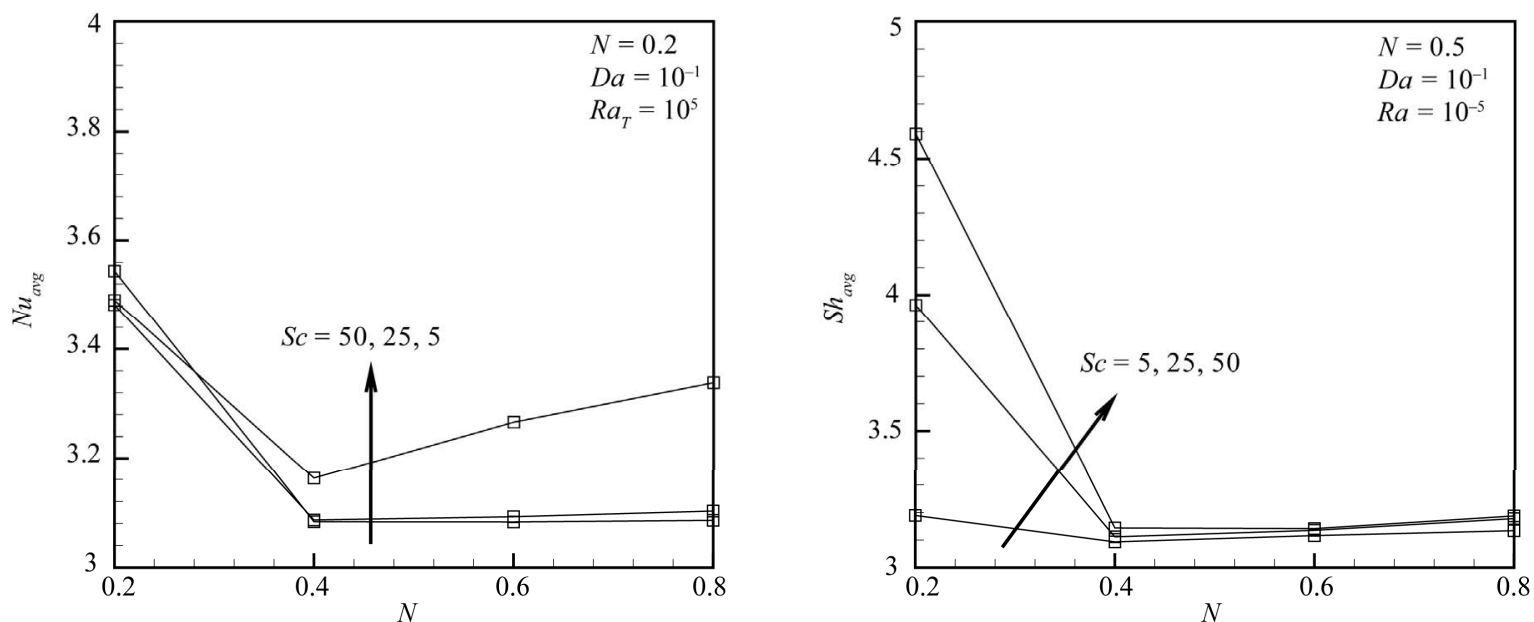

Figure 6. Average Nusselt number and Sherwood number for Sc Vs $N$.

sity maximum region. For such a situation, the dual cell structure inhibits the direct convective transfer of energy from one cell to another. This phenomenon results essentially from the inversion of the fluid density at $4^{\circ} \mathrm{C}$ and is one of its most significant effects on the mechanism of heat transfer by convection of water within the cavity.

\section{Conclusions}

Numerical computations are performed to study natural convection heat and mass transfer in a porous enclosure containing water near its density maximum. It is observed that the density inversion leaves strong effects on fluid flow, heat and mass transfer due to the formation of bi-cellular stature. The heat and mass transfer rate behaves nonlinearly with density inversion parameter and Schmidt number. The heat and mass transfer rates are found to decrease with decreasing thermal Rayleigh number. At the onset of convection dominant mode, the temperature contour lines get compressed toward the side walls and they tend to get deformed towards the upward direction.

\section{References}

[1] D. A. Nield and A. Bejan, "Convection in Porous Media (Third Edition)," Springer-Verlag, New York, 2006.

[2] K. Vafai, "Handbook of Porous Media," 2nd Edition, Marcel Dekker Inc., New York, 2005. doi:10.1201/9780415876384

[3] F. Alavyoon, "On Natural Convection in Vertical Porous Enclosures Due to Prescribed Fluxes of Heat and Mass Transfer at the Vertical Boundaries," International Journal of Heat and Mass Transfer, Vol. 36, No. 10, 1993, pp. 2479-2498. doi:10.1016/S0017-9310(05)80188-7

[4] M. Mamou, P. Vasseur and E. Bilgen, "Multiple Solution for Double-Diffusive Convection on a Vertical Porous Enclosure," International Journal of Heat and Mass Transfer, Vol. 38, No. 10, 1995, pp. 1787-1798. 
doi:10.1016/0017-9310(94)00301-B

[5] M. Nishimura, M. Wakamatsu and A. M. Morega, "Oscillatory Double-Diffusive Convection in a Rectangular Enclosure with Combined Horizontal Temperature and Concentration Gradients," International Journal of Heat and Mass Transfer, Vol. 41, No. 11, 1998, pp. 1601-1611. doi:10.1016/S0017-9310(97)00271-8

[6] I. Sezai and A. A. Mohamad, "Three-Dimensional Double-Diffusive Convection in a Porous Cubic Enclosure Due to Opposing Gradients of Temperature and Concentration," The Journal of Fluid Mechanics, Vol. 400, 1999, pp.333-353. doi:10.1017/S0022112099006540

[7] M. W. Nansteel, K. Medjani and D. S. Lin, "Natural convection of Water near Its Density Maximum in Ractangular Enclosure: Low Rayleigh Number Calculations," Physics of Fluids, Vol. 30, No. 2, 1987, pp. 312-317. doi: $10.1063 / 1.866379$

[8] A. Mahidjiba, L. Robillard and P. Vasseur, "On Set of Convection in a Horizontal Anisotropic," International Communications in Heat and Mass Transfer, Vol. 27, No. 6, 2000, pp. 765-774. doi:10.1016/S0735-1933(00)00157-3
[9] R. Bennacer, A. Tobbal, H. Beji and P. Vasseur, "Double Diffusive Convection in a Vertical Enclosure Filled with Anisotropic Porous Media," International Journal of Thermal Sciences, Vol. 40, No. 1, 2001, pp. 30-41. doi:10.1016/S1290-0729(00)01185-6

[10] M. Muthtamilselvan, M. K. Das and P. Kandaswamy, "Convection in a Lid-Driven Heat Generating Porous Cavity with Alternative Thermal Boundary Conditions," Transport in Porous Media, Vol. 82, No. 2, 2010, pp. 337-346. doi:10.1007/s11242-009-9429-7

[11] S. V. Patankar, "Numerical Heat Transfer and Fluid Flow," Hemisphere, Washington DC, 1980.

[12] T. Hayase, J. A. C. Humphrey and R. Grief, "A Consistently Formulated QUICK Scheme for Fast and Stable Convergence Using Finite-Volume Iterative Procedures," Journal of Computational Physics, Vol. 98, No. 1, 1992, pp. 108-118. doi:10.1016/0021-9991(92)90177-Z

[13] P. Kandaswamy, M. Muthtamilselvan and J. Lee, "Prandtl Number Effects on Mixed Convection in a LidDriven Porous Cavity," Journal of Porous Media, Vol. 11, 2008, pp. 791-801. doi:10.1615/JPorMedia.v11.i8.70 


\section{Nomenclature}

\begin{tabular}{|c|c|}
\hline$C$ & dimensional concentration \\
\hline$D a$ & Darcy number \\
\hline$G$ & gravitational acceleration \\
\hline$G r$ & Grashof number \\
\hline$L$ & enclosure length \\
\hline$K_{T}$ & thermal diffusion ratio \\
\hline$K$ & effective thermal conductivity of the porous medium \\
\hline $\mathrm{Nu}$ & Nusslet number \\
\hline$N u_{\text {avg }}$ & average Nusslet number \\
\hline$P$ & Pressure \\
\hline $\operatorname{Pr}$ & Prandtl number \\
\hline$R$ & dimensional density inversion parameter \\
\hline$R a_{c}$ & solute Rayleigh number \\
\hline$R a_{T}$ & thermal Rayleigh number \\
\hline$S c$ & Schmidt number \\
\hline$S h$ & Sherwood number \\
\hline$S h_{\text {avg }}$ & average Sherwood number \\
\hline$T$ & dimensionless temperature \\
\hline$U, V$ & dimensionless velocities in $X$ - and $Y$-direction \\
\hline$u, v$ & velocities in $x$ - and $y$-direction \\
\hline$X, Y$ & dimensionless Cartesian coordinates \\
\hline$x, y$ & Cartesian coordinates \\
\hline \multicolumn{2}{|c|}{ Greek symbols } \\
\hline$\alpha$ & effective thermal diffusivity of porous medium, $\mathrm{m}^{2} \cdot \mathrm{s}^{-1}$ \\
\hline$\beta_{T}$ & coefficient of thermal expansion, $\mathrm{K}^{-1}$ \\
\hline$\beta_{c}$ & coefficient of solute expansion \\
\hline$\Delta \theta$ & temperature difference \\
\hline$\theta$ & temperature, ${ }^{\circ} \mathrm{C}$ \\
\hline$K$ & permeability of porous medium, $\mathrm{m}^{2}$ \\
\hline$\mu$ & effective dynamic viscosity \\
\hline$v$ & effective kinematic viscosity \\
\hline$\rho$ & fluid density, $\mathrm{kg} \cdot \mathrm{m}^{-3}$ \\
\hline$\varepsilon$ & Porosity \\
\hline$\tau$ & dimensionless time \\
\hline \multicolumn{2}{|c|}{ Subscripts } \\
\hline$A v g$ & Average \\
\hline$C$ & cold wall \\
\hline$H$ & hot wall \\
\hline
\end{tabular}

